\title{
Investigation of the Velocity Factor in a Rotational Dynamic Microfiltration System
}

\author{
Marke, Henrik S.; Breil, Martin P.; Hansen, Ernst Broberg; Pinelo, Manuel; Krühne, Ulrich
}

Published in:

Separation and Purification Technology

Link to article, DOI:

10.1016/j.seppur.2019.03.012

Publication date:

2019

Document Version

Peer reviewed version

Link back to DTU Orbit

Citation (APA):

Marke, H. S., Breil, M. P., Hansen, E. B., Pinelo, M., \& Krühne, U. (2019). Investigation of the Velocity Factor in a Rotational Dynamic Microfiltration System. Separation and Purification Technology, 220, 69-77. https://doi.org/10.1016/j.seppur.2019.03.012

\section{General rights}

Copyright and moral rights for the publications made accessible in the public portal are retained by the authors and/or other copyright owners and it is a condition of accessing publications that users recognise and abide by the legal requirements associated with these rights.

- Users may download and print one copy of any publication from the public portal for the purpose of private study or research.

- You may not further distribute the material or use it for any profit-making activity or commercial gain

- You may freely distribute the URL identifying the publication in the public portal 


\section{Accepted Manuscript}

Investigation of the Velocity Factor in a Rotational Dynamic Microfiltration System

Henrik S. Marke, Martin P. Breil, Ernst Broberg Hansen, Manuel Pinelo, Ulrich

Krühne

PII:

S1383-5866(18)33946-7

DOI:

https://doi.org/10.1016/j.seppur.2019.03.012

Reference:

SEPPUR 15379

To appear in:

Separation and Purification Technology

Received Date: $\quad 9$ November 2018

Revised Date: $\quad 1$ March 2019

Accepted Date: $\quad 4$ March 2019

Please cite this article as: H.S. Marke, M.P. Breil, E. Broberg Hansen, M. Pinelo, U. Krühne, Investigation of the Velocity Factor in a Rotational Dynamic Microfiltration System, Separation and Purification Technology (2019), doi: https://doi.org/10.1016/j.seppur.2019.03.012

This is a PDF file of an unedited manuscript that has been accepted for publication. As a service to our customers we are providing this early version of the manuscript. The manuscript will undergo copyediting, typesetting, and review of the resulting proof before it is published in its final form. Please note that during the production process errors may be discovered which could affect the content, and all legal disclaimers that apply to the journal pertain. 


\title{
Investigation of the Velocity Factor in a
}

\section{Rotational Dynamic Microfiltration System}

\author{
Henrik S. Marke ${ }^{(a, b)}$, Martin P. Breil ${ }^{(b)}$, Ernst Broberg Hansen ${ }^{(b)}$, Manuel Pinelo ${ }^{(a)}$, Ulrich Krühne ${ }^{(a, 1)}$ \\ a) Department of Chemical and Biochemical Engineering, Technical University of Denmark, Søltofts Plads 229, 2800 Kgs. Lyngby, Denmark \\ b) Novo Nordisk A/S, Bagsværd, Denmark
}

\begin{abstract}
:
This work aims to advance the understanding of rotational dynamic filtration by investigation of the counter pressure generated by rotation. The counter pressure was experimentally evaluated with a series of experiments. The experimental conditions were varied by using mixtures of water/ethanol, different module geometries, various permeate flows, and rotational speeds for a total of 416 experiments. In addition computational fluid dynamic (CFD) simulations of the experimental setup were performed, leading to a better understanding of the experimental results. The results were assessed with a simplifying engineering model. It was demonstrated, that the experimental counter pressure diverges from the theoretical values. The experimental results suggest that a velocity factor should be included in the equation for counter pressure in rotating membrane systems. This finding was supported by evaluation of the CFD simulations, where the velocity factor also was found to diverge from theory. The study of module geometries also indicates that there is a small impact from geometry of the housing on the counter pressure, suggesting a need to determine the velocity factor for each system. This study provides new information on the influence of geometry and operational conditions on the counter pressure in rotating systems, a key parameter in the design and operation of such units.
\end{abstract}

Keywords: Membrane filtration; CFD; Dynamic Filtration; Velocity Factor; Counter Pressure

\section{Introduction}

Membrane filtration has shown superior efficiency in many separation applications, such as lower energy demand in desalination of water by reversed osmosis compared to distillation [1]. However, for some applications membrane separation is not suitable. One of the factors limiting the implementation of membrane filtration in new applications is fouling. For many separation applications, membranes foul too easily for the process to be economically sound [2]. It has been repeatedly shown, that there is a strong link between the shear force at the membrane surface and the capacity of a membrane to resist fouling [3]. Crossflow filtration is an example of a technique that promotes filtration performance by shear forces. Here the shear is generated by the flow of the solvent over the membrane surface. The technique is however

\footnotetext{
${ }^{1}$ Corresponding author: Email: ulkr@kt.dtu.dk
} 
limited by the pressure drop generated at high flow rates and the stress on solids and equipment by pump work.

Dynamic filtration, an alternative to crossflow filtration, has gained attention as a technology that can enable filtration of heavy fouling solutions as very high shear force is attainable. In dynamic filtration, the generation of shear force is decoupled from the flow generated by the feed pump. This gives the dynamic filtration system a distinct advantage over regular crossflow filtration systems, as a higher shear force is achievable, without excessive energy consumption [4]. Different types of dynamic filtration have been described, such as vibrating systems [5] or rotating systems, where either the membrane or an impeller rotates to keep the membrane clean [6]. However the higher shear rate comes at a cost; in rotational dynamic filtration the motion of the membrane gives rise to a centrifugal force, working against the driving pressure of the filtration. This counter force hampers the filtration, and has to be accounted for when describing the filtration.

In its most simple form the impact of the rotation speed $(\omega)$ on the flux $(J)$ can be described as:

$$
J=a\left(\Delta P-b \omega^{2}\right) \#(1)
$$

Where $\Delta P$ is the pressure drop between the feed pressure and the permeate pressure, $a$ the solvent permeability, and $b$ an empirical coefficient [7]. In Equation 1, the parameter $b$ links the rotational speed to the counter pressure $\left(P_{c}\right)$ as:

$$
P_{c}=b \omega^{2} \#(2)
$$

G. Belfort et al. [7] determined the value to be $b=1.8 \times 10^{4} \mathrm{~kg} / \mathrm{m}$, for their system with a rotating annular membrane. For a different type of systems, the value will be different. For instance in dynamic systems, where an impeller moves over the membrane to generate the shear forces, the expression for $b$ has been reported as [8]:

$$
b=\frac{\rho}{4}\left(R k_{r}\right)^{2} \#(3)
$$

Where $\rho$ is the density of the liquid, $R$ the outer radius of the rotor, and $k_{r}$ the velocity factor, an empirical constant indicating the how effectively the power from the rotor is transferred to the membrane surface. For these systems the values for $k_{r}$ are generally less than 1. For smooth discs $k_{r}$ are typical found to be between 0.30 and 0.45 . For systems with vanes or blades values up to 0.8 have been reported $[9,10]$.

For systems where the membrane itself is rotating, as the one studied in this work, $b$ is ordinarily determined from the system geometry according to the following expression [4]:

$$
b=\frac{\rho}{4}\left(r_{1}^{2}+r_{2}^{2}\right) \#(4)
$$


Where $r_{1}$ and $r_{2}$ is the inner and outer radius of the rotating membrane, respectively. It is worth noticing that the expression is fully defined, meaning all values are easily measured, the equation is without any empirical constants.

The understanding of counter pressure has been incorporated into studies of dynamic filtration systems. As an example, Dal-Cin et al. [11] accounts for the counter pressure $\left(P_{c}\right)$ by correcting the measured flux by the ratio of the operational pressure $(\Delta P)$ over the effective pressure:

$$
J_{V C}=J_{V} \frac{\Delta P}{\Delta P-P_{c}} \#(5)
$$

This is done in order to obtain the corrected flux $\left(J_{V C}\right)$ based on the measured flux $\left(J_{V}\right)$. The corrected flux is a theoretical abstraction, allowing results obtained at different rotational speeds to be compared. Another way to account for the counter pressure, is to establish the theoretical TMP of the system. This was done in both the work of P. Meyer et al. [9] and W. Zhang et al. [12] by calculating:

$$
T M P=\Delta P-P_{C} \#(6)
$$

By expressing all data as a function of TMP vs flux, $P_{c}$ can be eliminated from the experimental evaluation. By taking $P_{c}$ into account, the flux from different filtration conditions are comparable. This enables a better insight into membrane fouling, as the pressure resulting from membrane rotation is separated from the pressure related to fouling. It is therefore essential to know the magnitude of $b$ from Equation 1 to draw valid conclusion form experiments.

Evaluation of the parameter $b$ has been done before for systems with an overhead rotor, but for systems with rotating membranes, only one study was found. Here Y. Taamneh and S. Ripperger [13] found a significant derivation from Equation 4. This conflicts with the usual approach, where Equation 4 is assumed valid. Therefore it was decided to explore the $b$ parameter for systems with rotating membranes.

The parameter $b$ was investigated by a set of equations, an engineering model describing the dynamic filtration system in terms of simple mechanisms. The proposed engineering model was tested experimentally and evaluated to demonstrate its validity. The engineering model was also used to define which features to include in the CFD simulation of the experimental setup. The CFD simulation was evaluated using the same approach as the evaluation of the experimental system. The results from both the experimental setup and the CFD model, were compared and analyzed in order to gain an understanding of the $b$ parameter.

\subsection{Model introduction}

A generic membrane filtration system can be described with a model linking the flux through the membrane to the driving pressure. One such model is the Darcy's law, as described in [14].

$$
J=\frac{T M P}{\mu R_{m}} \#(7)
$$


Where $J$ is the flux through the membrane, TMP, the transmembrane pressure, $\mu$ the dynamic viscosity, and $R_{m}$ the resistance to flux in the membrane system. TMP in Darcy's law is determined as the pressure difference between the pressure at the membrane surface and the pressure at the permeate side of the membrane. Equation 7 is only valid for solvent systems, i.e. in the absence of concentration polarization and fouling.

Reiterating, for rotational dynamic filtration, the operating pressure $(\Delta P)$, i.e. the pressure difference between inlet pressure and permeate pressure is not equal to the TMP, as the rotation of the membrane generates a counter pressure $\left(P_{c}\right)$, as shown in [9]. The pressure drop associated with the rotation of the membrane depends on the rotational speed of the membrane $(\omega)$, the liquid density $(\rho)$, and the geometry of the membrane $(r)$ and is given as:

$$
P_{c}=b \omega^{2}=k_{1}^{2} \frac{\rho}{4}\left(r_{1}^{2}+r_{2}^{2}\right) \omega^{2} \#(8)
$$

In Equation 8 the parameter $k_{1}$ has been included in order to quantify the deviation from ideality. It is similar to the velocity factor, and here it indicates the efficiency, by which the rotation of the membrane generates counter pressure. For these systems the velocity factor is usually absent in the expression, i.e. the expected value is unity $[4,11,15]$. The proposed engineering model for $\Delta P$ is given as the sum of $P_{c}$ and TMP:

$$
\Delta P=P_{c}+T M P=k_{1}^{2} \frac{\rho}{4} \omega^{2}\left(r_{1}^{2}+r_{2}^{2}\right)+J \mu R_{m} \#(9)
$$

In the experimental section the results will be evaluated using the proposed engineering model. Equation 9 has to be modified such that the parameters corresponds to the values measured in the experiment. The Flux can be calculated from the volumetric permeate flow rate $(F)$, by accounting for the membrane area $(A)$, as $J \cdot A=F$. This allowed for the following expression to be obtained:

$$
\Delta P=k_{1}^{2} \frac{\rho}{4} \omega^{2}\left(r_{\text {inner }}^{2}+r_{\text {outer }}^{2}\right)+k_{2} \mu J \#(10)
$$

Where $k_{1}$ the velocity factor, and $k_{2}$ the membrane resistance. As $R_{m}$ is an inherent property for the membrane, $k_{2}$ is expected to be independent of the solvent and the rotation velocity of the membrane, furthermore it should be constant over time. By conducting experiments with a stationery membrane $(\omega=$ 0 ) the effects of counter pressure can be removed. This simplifies the assessment of $k_{2}$, as $P_{C}$ from Equation 9 can be omitted. To get the interaction between $J$ and $k_{2}$ the equation is further simplified by normalizing with the viscosity:

$$
\frac{\Delta P}{\mu}=J \cdot k_{2} \#(11)
$$

\subsection{Introduction to the CFD model}

In addition to the experimental work, a CFD model of the experimental installation was established. In order to evaluate the validity of the CFD model, the experimental data was compared to the simulations. The CFD 
simulation were performed in ANSYS CFX ver. 17.2 and the evaluation of the simulations were performed in ANSYS CFD-Post ver. 17.2.

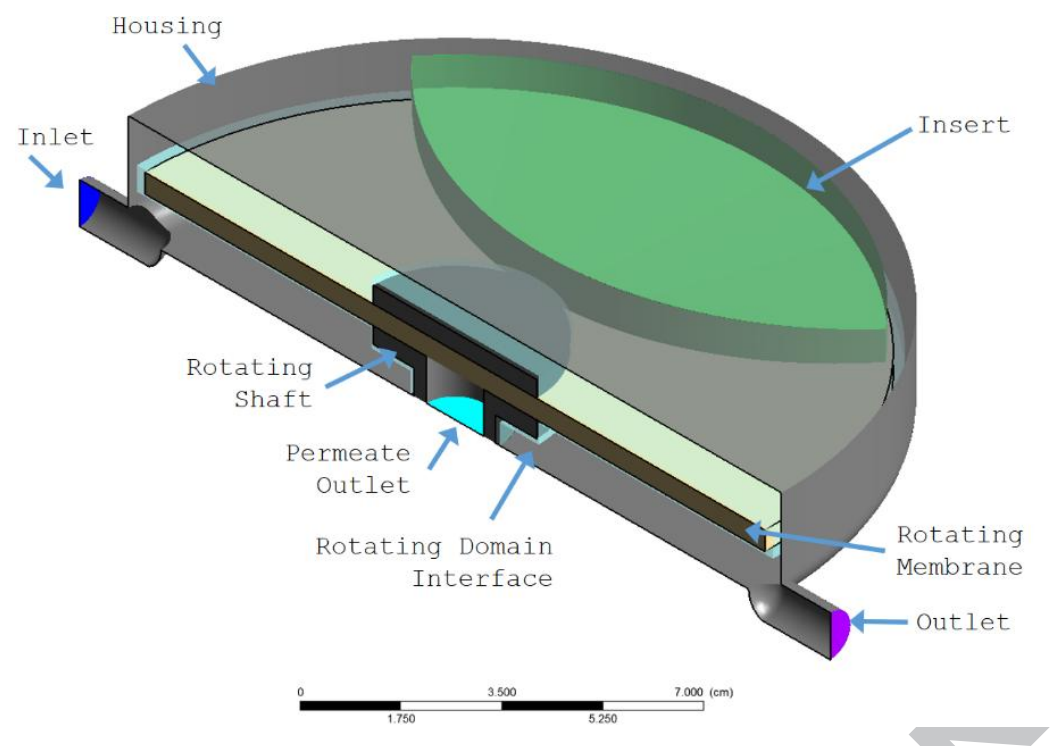

Figure 1: Scaled Drawing of the simulated geometry

Half Plane of the simulated geometry. Features; Dark Blue: Inlet. Purple: Outlet. Light Grey: Housing, stationary walls. Dark Grey: Shaft, rotating walls. Light Blue: transition domain interface. Orange: Rotating membrane. Teal: Permeate outlet.

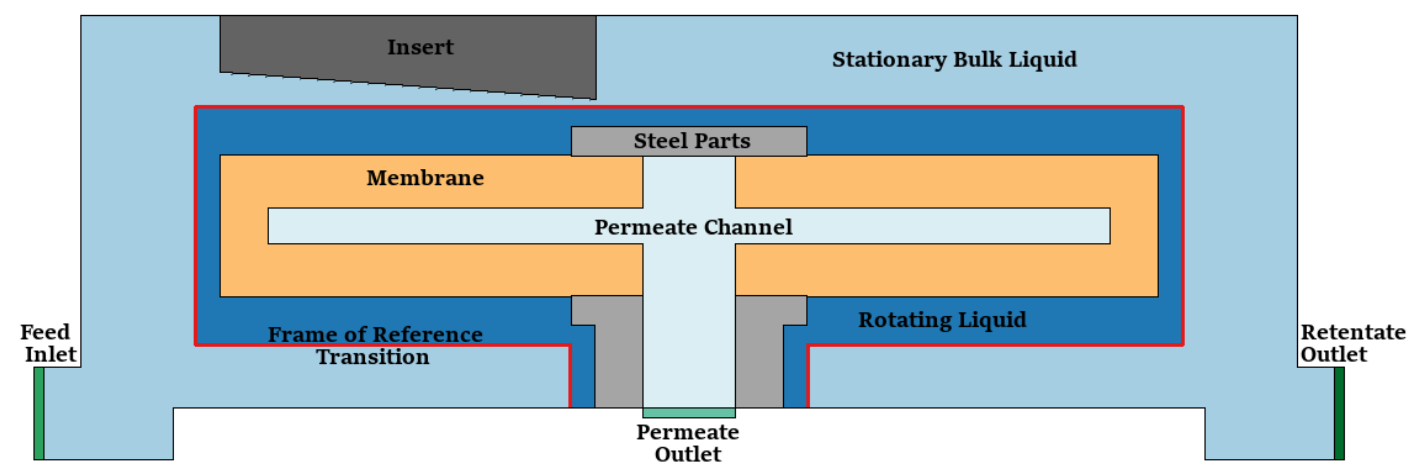

Figure 2: Conceptual sketch of the simulated geometry

Outlining the conceptual parts in the simulated geometry. In green colors: Inlet and outlets. In blue colors:

Free flowing liquid domains. In yellow: The membrane. In grey: Steel parts. The red line denotes the transition from a stationary frame of reference to a rotating frame of reference.

A sketch of the liquid geometry was generated in SolidWorks based on the physical dimensions of the experimental unit. In Figure 1 the geometry is shown to scale with the important features highlighted. To better show how the parts of the geometry interact, a conceptual sketch of the geometry is given in Figure 2 . The liquid geometry was meshed with a combination of unstructured meshes for the bulk liquid and structured meshes for the liquid in and around the membrane. The frame of reference and number of elements for each domain is listed in Table 1. 
Table 1: Mesh Elements for the parts of the CFD model

The frame of reference, mesh type, and number of elements in the different parts in the CFD model iviesn rart rrame or kererence Iviesn I ype \# or ciements

\begin{tabular}{rrrr}
\hline Membrane Domain & Rotating & Structured & 390456 \\
Near membrane Liquid Domain & Rotating & Structured & 335308 \\
Permeate Domain & Rotating & Structured & 390456 \\
Liquid Domain, without insert & Stationary & Unstructured & 1413849 \\
Liquid Domain, with insert & Stationary & Unstructured & 1309803 \\
Total without insert & & & 2530069 \\
Total with insert & Combination & Combination & 2426023
\end{tabular}

Important for the CFD model is the simulation of the membrane, and its ability to mimic the properties of a real membrane. The membrane and liquid near the surface was meshed as structured domains, with the membrane domain model as a porous domain. The resistance to flow in the membrane was included as a momentum loss in the source term of the Navier-Stokes equation. At low speed the flow through the membrane is laminar and the momentum loss can be found using Darcy's law, thus it depends linearly on the flow rate. The total momentum loss $\left(S_{M, i}\right)$ was assumed isotropic and given by the following expression:

$$
S_{M, i}=-\frac{\mu}{K_{\text {perm }}} U_{i} \#(12)
$$

Here $U_{i}$ is the velocity in direction $i, K_{\text {perm }}$ the Darcy's loss coefficient, equivalent to $R_{m}$.

The structure of the real membrane is complex. It has an intricate support structure, with internal channels to drain permeate. The surface is coved by a skin layer, with a very small pore size. To replicate the characteristics of the membrane, the porosity was implemented as a compounded mathematical expression, where the porosity $(\varepsilon)$ of the support layer was set independently of the skin layer. It was chosen to model the internal composition as consisting of three distinct regions, an skin layer, with $\varepsilon=0.025$, a larger support layer with $\varepsilon=0.05$, and a channel region where liquid flows unimpeded, i.e. $\varepsilon=1$, as seen in Figure 3 . To model the increased resistance in the skin layer, $K_{\text {perm }}$ was estimated as having an exponential correlation with the porosity according to Equation 13:

$$
K_{\text {perm }}=\exp \left(\frac{\varepsilon-1}{\alpha}\right) \#
$$




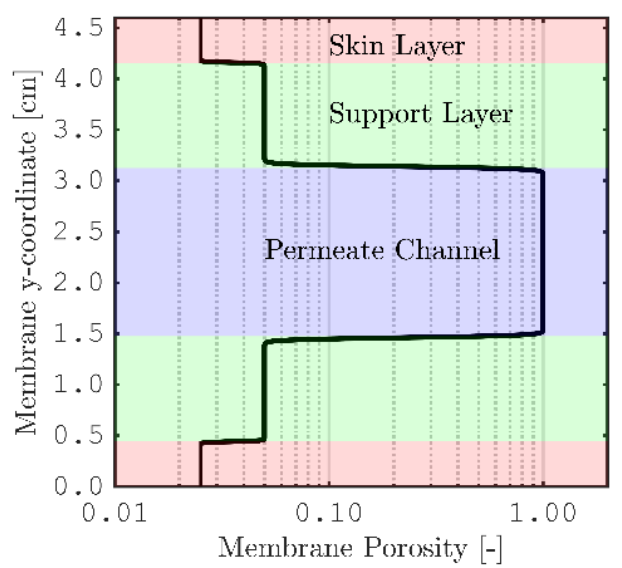

Figure 3: Modelled Structure and Porosity of the Membrane The internal structure of the membrane in the simulation seen from the side. X-axis scale with log, to better show the porosity at the skin layer. Black Line: Porosity in the model. Red Area: Skin layer, Green Area: Support layer, Blue Area: Permeate Channel.

The thickness of the surface layer was defined to $0.443 \mathrm{~mm}$, the supporting layer to $1.03 \mathrm{~mm}$, and the channel to $1.65 \mathrm{~mm}$, for a total thickness of $4.60 \mathrm{~mm}$. The value of $\alpha$ in Equation 13 was adjusted, such that the overall resistance in the system was comparable to the experimental system. This resulted in a value of $\alpha=0.0315$.

The model was separated into two frames of references, a stationary and a rotating frame. The stationary frame encompasses the housing domain. This domain describes the behavior of the bulk liquid, and contains the feed inlet and the retentate outlet. The rotating frame encompasses the membrane domain, the permeate domain, and a transition fluid domain between the membrane surface and the bulk liquid. The membrane was defined as a porous domain with a rotating frame. The transition domain was included in the rotating frame, to isolate the boundary between the porous and liquid domain, from the change in reference frame. This reduces the numerical errors associated with the change in frame of reference at the important transition from porous media to free flowing liquid. All simulation was done as steady state simulations.

For the CFD simulation the boundary conditions were set to mirror the experimental conditions as closely as possible. This means a fixed inlet mass flow of $298 \mathrm{~kg} / \mathrm{h} / \mathrm{m}^{2}$, and a retentate outlet flow of $178 \mathrm{~kg} / \mathrm{h} / \mathrm{m} 2$, corresponding to $60 \%$ of the feed flow. The permeate outlet was defined as an opening with a constant absolute pressure of 1 bar. This allowed for the CFD simulation to find the feed pressure needed to equalize the mass balance. All the walls in the model were modelled with a no-slip condition. The static walls in the rotating domain were additionally defined as counter rotating, such that they become stationary. 
A theoretical estimate for the Reynolds number, can be made based on water viscosity, the distance between membrane and module house as critical length. The Reynolds number, at the outer edge of the disc depends on the rotational speed as: $R e=\omega \cdot 526 R P M^{-1}$. The rotational speeds used in this work gives Reynolds number sufficiently high, that turbulence is expected in the module. For the liquid phase, Shear Stress Transport (SST) model was chosen to model the turbulence, due to its superior resolution of the flow near to the walls.

In order to ensure the quality of the mesh two controls were performed. Mesh independency was studied by constructing a refined mesh, containing $30 \%$ more elements, than the mesh employed for the regular simulations. Comparison at 190 RPM showed no difference in evaluated $\Delta P$ with the refined mesh. Additionally $\mathrm{Y}+$ value at different positions along the membrane surface was evaluated. For the SST model to be correct a $\mathrm{Y}+$ value below 5 is recommended for the first note element over the surface. In Figure 4 , the actual values for $\mathrm{Y}+$ is shown, for the fastest (worst) simulated case, i.e. $\omega=1150$ RPM. For all locations on the membrane surface the $Y+$ value is $<3$, fulfilling the requirement.

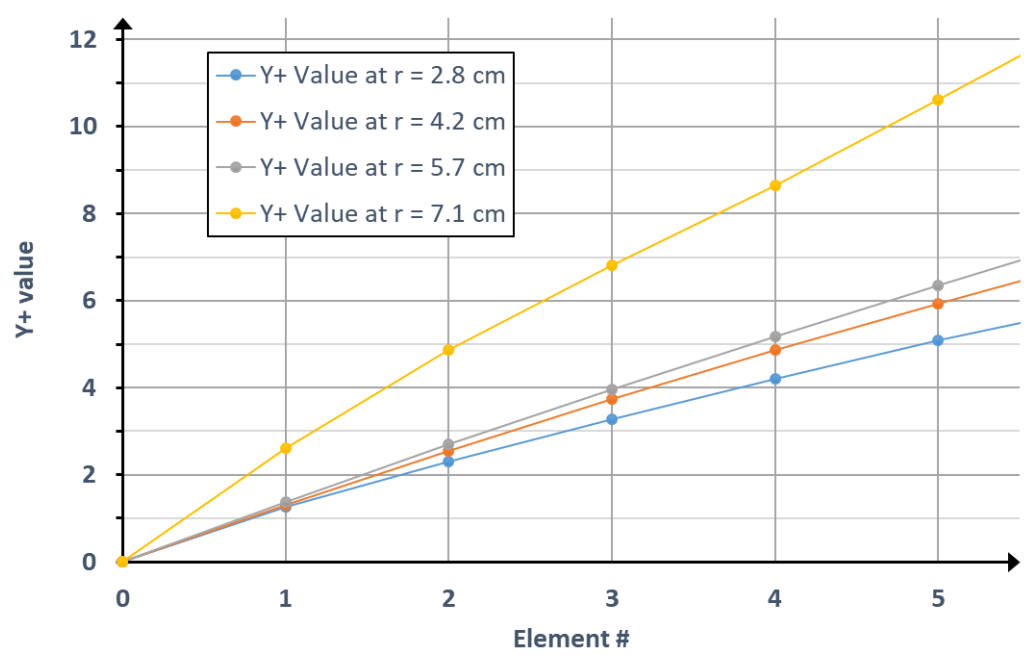

Figure 4: $\mathbf{Y}+$ values in the liquid domain over the membrane surface The $Y+$ value calculated for the spinning disc of the simulations, at 1150 RPM. Four different positions shown, to show the change from the center of the membrane to the edge. For all cases, the $Y+$ is below 5 


\section{Experimental}

\subsection{Equipment}

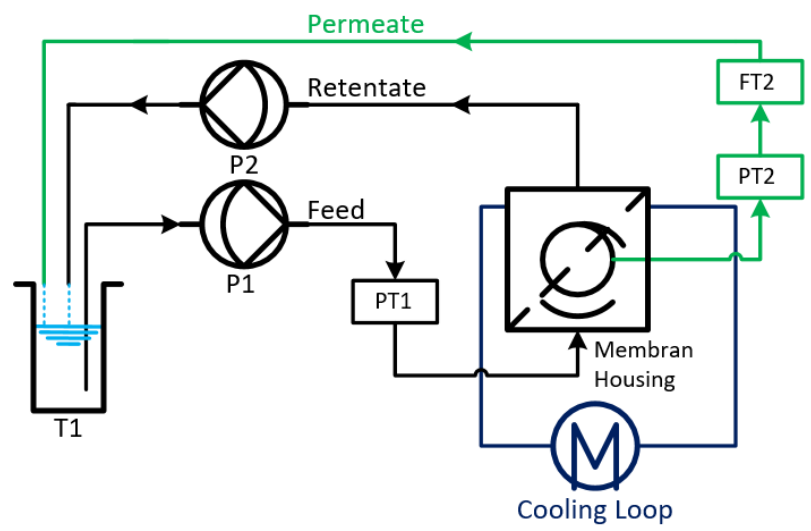

Figure 5: Schematic diagram of experimental apparatus.

Sketch of the experimental installation. Named components: P1 Feed Pump, P2

Retentate Pump, PT1 Feed Pressure Transmitter, PT2 Permeate Pressure showed in Figure 5. At the core of Transmitter. FT2 Permeate Flow Meter, T1 Reservoir tank nouses the dynamic membrane. The feed liquid was drawn from the reservoir tank (T1), and circulated through the system. Both retentate and permeate were returned to the reservoir tank to ensure constant feed composition. The permeate flow (FT2) was controlled by the difference in pump speed between the feed pump (P1) and retentate pump (P2) both peristaltic pumps (Watson-Marlow 323Du), and measured by a volumetric flowmeter (omega, FLR1008-D). This ensured a stable flux for each experimental setting. The house pressure (PT1) was measured (JUMO MIDAS), immediately before the inlet to the membrane house, its measurement reflecting the pressure in the house. The permeate pressure (PT2) was also measured (JUMO MIDAS). The operating pressure $(\Delta P)$ was defined as the difference between the house pressures and the permeate pressure. To control the experiment and to collect the transmitter data the installation was connected to a computer via a LabJack T7 module and the data was recorded with help of LabVIEW (ver. 2017 SP1). The temperature in the membrane housing, and thereby in the experiment, was kept constant $\left(20^{\circ} \mathrm{C}\right)$ using a water bath connected to a cooling jacket around the membrane housing.

The membrane chamber has a mount for metal inserts, designed to imitate the effects of additional discs overlapping the rotating disc. Having overlapping spinning discs have been shown to increase filtration performance [16]. The metal inserts provided an opportunity to study the effects of a disturbance in the bulk flow. In Figure 5 the geometry with and without the insert are shown, a similar insert is present at the front plate (not shown). Experiments have been done with and without the insert, in order to evaluate how $\Delta P$ is affected by disturbed flow paths. 


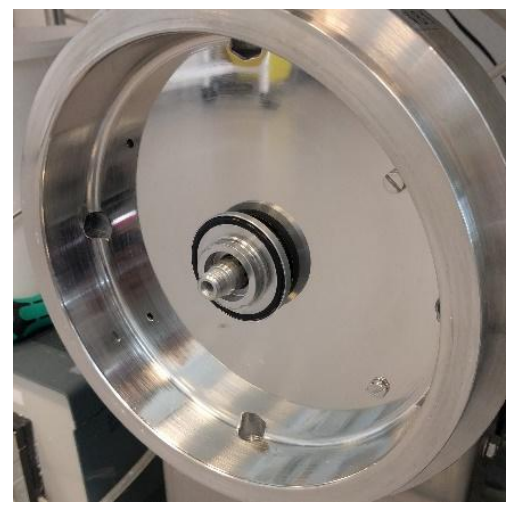

A: Membrane module without insert

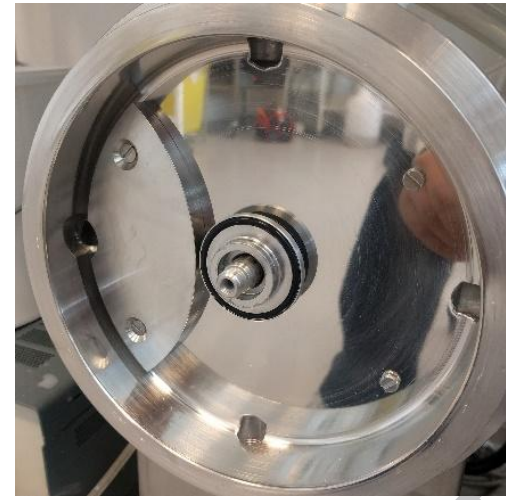

B: Membrane module with insert

Figure 6: Overview of membrane module geometry

The position and size of the turbulence inducing insert shown in the module housing. A similar insert was installed on the front of the module house, not shown in the figure

\subsection{Membranes}

The membranes used in the experiments were circular ceramic $\left(\mathrm{Al}_{2} \mathrm{O}_{3}\right)$ membranes (KERAFOL) with an outer diameter of $d_{\text {outer }}=152 \mathrm{~mm}$, a shaft diameter of $d_{\text {shaft }}=25.5 \mathrm{~mm}$, and a thickness of $4.6 \mathrm{~mm}$. Due to fittings, the inner working diameter of the membrane was $d_{\text {inner }}=38 \mathrm{~mm}$. Before each experiment, the membrane and the installation were cleaned with a cycle containing a sodium hydroxide $\left(0.5 \mathrm{w} / \mathrm{w} \%\right.$, at $\left.50{ }^{\circ} \mathrm{C}\right)$ soak for one hour and a nitric acid $(0.5 \mathrm{w} / \mathrm{w} \%)$ wash. Membranes with two different pore sizes were used in the experiments. One with a pore size of $0.5 \mu \mathrm{m}$ and one with a pore size of $0.2 \mu \mathrm{m}$.

\subsection{Materials}

For the experiments purified-water and ethanol (70 v/v\%) was used. As only solvents was used, the membrane was expected to have no retention, curtailing any polarization in the experiment. The ethanol was added to the solvent to alter the viscosity. The ethanol water mixture was prepared shortly before use, in order to minimize evaporation. The resulting mixture had a realized ethanol concentration between $12 \mathrm{w} / \mathrm{w} \%$ and $17 \mathrm{w} / \mathrm{w} \%$. The viscosity of the ethanol water mixture was measured to be $1.53 \pm 0.06 \mathrm{cP}$, using a coneand-plate viscometer (Brookfield ${ }^{\mathrm{TM}}$, DV2T CPA-40Z).

\subsection{Experimental Conditions}

The experiments were conducted in series, within each series the experiments had the same conditions, i.e. constant geometry, membrane disc, and feed solvent. For each series the installation was stepped through a set of parametric values for the rotational speed and the permeate flux. For each set point, the system was allowed to equilibrate. After equilibration the effective permeate flow rate and operational pressure were recorded, with a data sampling rate of $15 \mathrm{~Hz}$. After a complete experimental series, the module was drained and cleaned. Then the experimental conditions were changed as needed, and a new experimental series were started. The conditions and parameter values of the experiments are shown in Table 2. The experiments are separated into two runs. The first run was done with 10 parameter sets, here the system was allowed six minutes for equilibration and two minutes for data collection. After the first experimental run, it was realized, 
that 6 minutes for equilibration was needlessly long time. The second run was therefore executed with one minute for equilibration and one minute for data collection. This meant 42 parameter sets could be included in the experimental series within the same timeframe. For both runs, the parameter list for rotational speeds contains the number zero twice, as the first set point, and as the last set point. This was done to enable evaluation of potential fouling within an experimental series.

Table 2: Experimental Conditions and parameter values

Overview of the experimental conditions and parameter values for the conducted experiments.

8 different conditions and 52 parameter sets give 416 unique experiments.

The parameter list for rotational speeds contains the zero, as the first and the last value, this enables evaluation of fouling within an experimental series.

\begin{tabular}{rrcc} 
Both runs & Geometry & 2 & Without inserts, With inserts \\
& Disc & 2 & $0.2 \mu \mathrm{m}, 0.5 \mu \mathrm{m}$ \\
\hline \multicolumn{2}{r}{ Permutations of Conditions: } & $\mathbf{8}$ & \\
Run & Parameter & \# of values & Experimental values \\
\hline $1^{\text {st }}$ run & Rotation Speed & 5 & $0,273,491,1091,0[\mathrm{RPM}]$ \\
& Permeate Flux & 2 & $86,171\left[\mathrm{~L} \mathrm{~h}^{-1} \mathrm{~m}^{-2}\right]$ \\
\hline $2^{\text {nd }}$ run & Rotation Speed & 7 & $0,273,491,682,900,1091,0[\mathrm{RPM}]$ \\
& Permeate Flux & 6 & $40,86,128,171,219,40\left[\mathrm{~L} \mathrm{~h}^{-1} \mathrm{~m}^{-2}\right]$ \\
\hline
\end{tabular}

\begin{tabular}{ccc}
\hline Permutation of parameters: & 52 \\
& & 416 \\
\hline Total experiments: & $52 \times 8$ & 416 \\
\hline
\end{tabular}




\section{Results and discussion}

\subsection{Typical Experimental Pressure Measurements}

For each parameter set, the collected steady state data had more than 800 discrete data points fluctuating around the average mean value. To facilitate the analytic work the arithmetic mean value and the standard deviation of each parameter set was calculated. The mean value was used, as the studied effect is independent of time. In Figure 7 and Figure 8 the results of a single experimental series are presented, showing the average results for 52 experimental set points. In Figure 7 a linear relationship between permeate flux and $\Delta P$ can be seen, this is related to Darcy's law. Likewise, in Figure 8 the nonlinear relationship between disc rotational speed and $\Delta P$ can be seen, this is linked to the counter pressure.
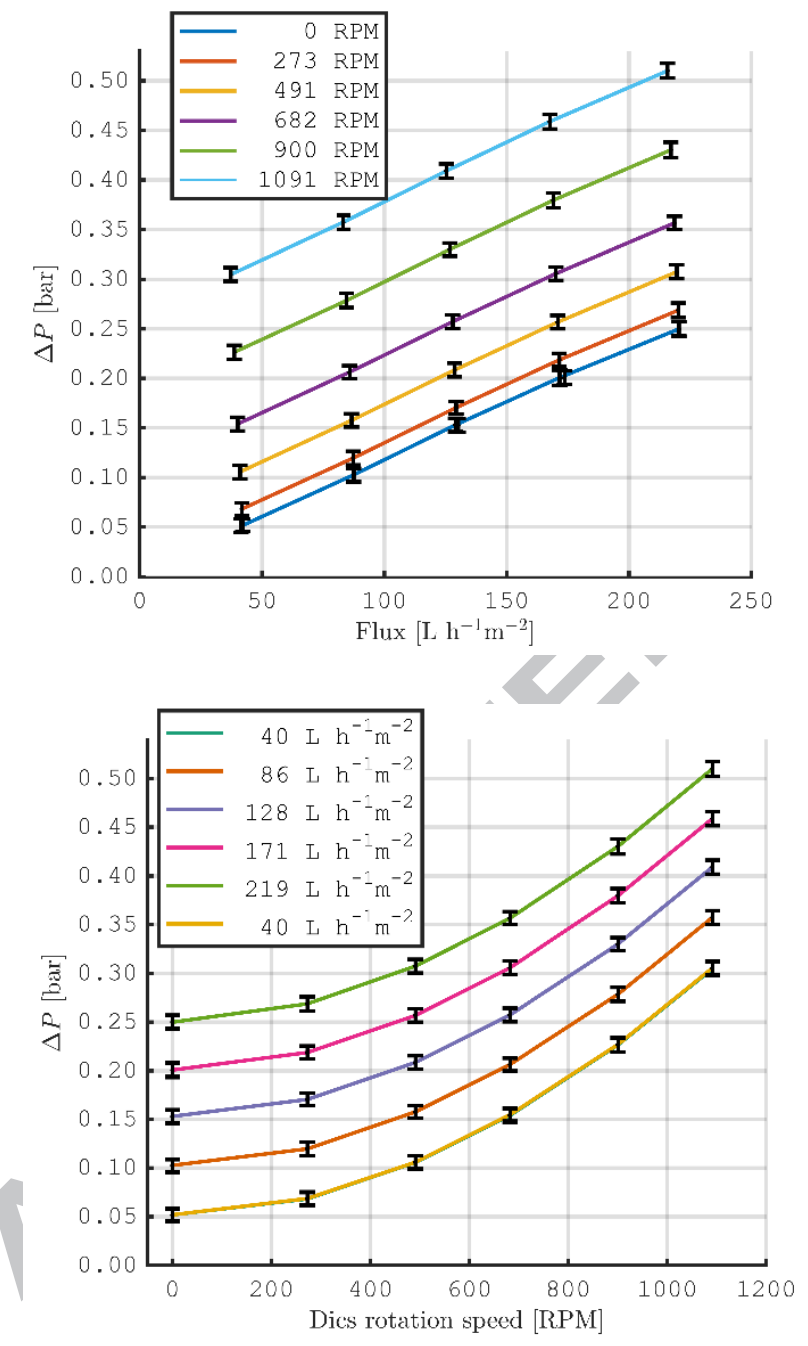

Figure 7: Experimental Pressure vs Permeate Flux Example of an experimental series from the $2^{\text {nd }}$ run of the conditions. The data was collected under the following conditions, solvent: Water, Disc: $0.2 \mu \mathrm{m}$, Geometry: without inserts. The vertical bars indicates standard deviation.

The color lines indicates experiments of similar disc rotational speed.

Figure 8: Experimental Pressure vs Disc rotational Speed Example of an experimental series from the $2^{\text {nd }}$ run of the conditions. The data was collected under the following conditions, solvent: Water, Disc: $0.2 \mu \mathrm{m}$, Geometry: without inserts. The vertical bars indicates standard deviation.

The color lines indicates experiments with similar permeate flux. The line labelled $40 \mathrm{~L} \mathrm{~h}^{-1} \mathrm{~m}^{-2}$ represent in reality two lines (green and yellow), which are perfectly overlapping. 


\subsection{Membrane Resistance}

By fitting the experimental data to Equation 11, the $k_{2}$ value for each set of conditions could be determined. In Figure 9 stationary data from four experimental series are shown as well as the fit of Equation 11. The four series were selected, as they all has the same membrane and all have the insert installed. As shown in the figure, $k_{2}$ and thereby the membrane resistance, is not a single value, but varies between each experimental condition. The values found for $k_{2}$ ranges from $3.77 \times 10^{5}\left[\mathrm{~m}^{-1}\right]$ to $1.19 \times 10^{6}\left[\mathrm{~m}^{-1}\right]$.

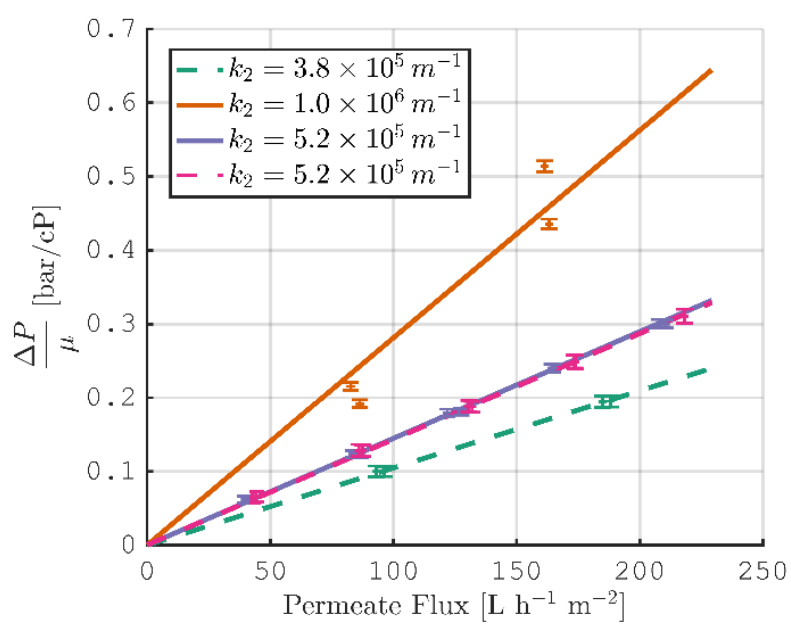

Figure 9: Normalized Pressure vs Permeate Flux for a stationary disc.

Pressure normalized over the viscosity of the liquid. Stationary disc data from 4 experimental series shown. Shared values: $0.2 \mu \mathrm{m}$ disc plus insert. Points are experimental values, vertical bars indicates standard deviation, and lines represent the fitted model. Solid lines: water, $\mu=1 \mathrm{cP}$. Dashed lines: Water/ethanol $\mu=1.5 \mathrm{cP}$. Slope equals $k_{2}$, according to Equation 11

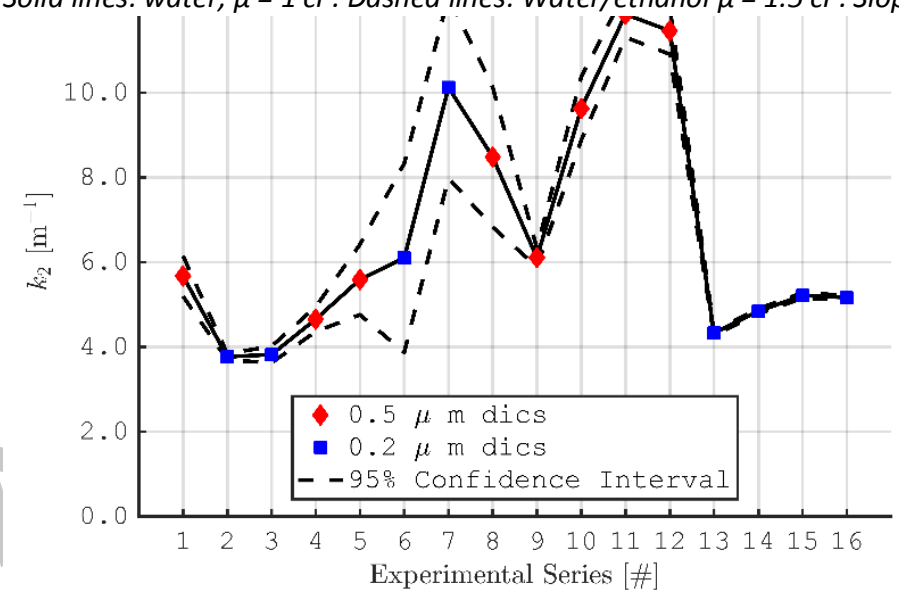

Figure 10: $\boldsymbol{k}_{2}$ vs Experimental series number

Showing the model parameter $k_{2}$ determined from experiments with stationary disc. $\$: 0.5 \mu \mathrm{m}$ disc. $\square: 0.2 \mu \mathrm{m}$ disc. The dashed lines represent the $95 \%$ confidence interval of the parameter. 
This procedure was applied to all experimental series, and the independent $k_{2}$ was determined. The computed $k_{2}$ values are given in Figure 10 with their $95 \%$ confidence interval. Before conducting the experiments it was expected that a homogeneous and constant $k_{2}$ would be obtained, since the membrane was consistently washed as indicated in the methods section between each experimental series. The range in determined $k_{2}$ values shows a variability in the membrane resistance not included in the model. Looking at the data from one experimental condition, exemplified in Figure 7, the variability is not observed within the experimental series. As it was ensured that the system reached steady state before the measurements were conducted, illustrated by the low standard deviation in the recorded data. The variation from one condition to the next can be significant, exemplified by the change in $k_{2}$ from series \#9 to \#12, which all used the 0.5 $\mu \mathrm{m}$ disc. Here the solvent was changed from water (\#9) to water/ethanol (\#10), then the module was open and the inserts removed (\#11), before the solvent was changed again, back to water (\#12). The determined $k_{2}$ values indicate, that it is necessary to distinguish between the experimental conditions, by using the specific $k_{2}$ value for each experimental condition. Based on the observations, that the changes in $k_{2}$, were associated with opening of the unit or exchange of solvent, it is therefore assumed, that either mechanical effects or a difference in resting days between experimental conditions, are responsible for the deviations of the values. This effect has been observed in other works, where the flux has been normalized with the initial flux, in order to make the ensuing experiments comparable $[14,15]$. This underlines the importance of measuring the initial membrane resistance before each experiment. Without the correction in $k_{2}$, any further evaluation of the experimental system would be flawed by the difference of initial resistance.

\subsection{Velocity Factor}

Using the determined $k_{2}$ corresponding to each experimental condition, the velocity factor $\left(k_{1}\right)$ can be studied. Unlike $k_{2}$ the velocity factor is expected to be independent of experimental series. Consequently it is possible to employ the complete data set to determine one value for $k_{1}$, if the individual $k_{2}$ are taken into consideration. Therefore was a revised version of Equation 10 used, where $i$ indicates the experimental series.

$$
\Delta P=k_{1}^{2} \frac{\rho}{4} \omega^{2}\left(r_{\text {inner }}^{2}+r_{\text {outer }}^{2}\right)+k_{2, i} \mu J \#(14)
$$

According to the prevalent representation of the counter pressure $k_{1}$ is expected to be 1 for systems as the one described in this work [6]. The validity of this value, can be tested by using $k_{1}=1$ in equation 14 and examining the residuals of the predicted $\Delta P$. In Figure 11 the residuals, calculated as model values over experimental values, sorted by the rotational speed of the membrane are plotted. If $k_{1}=1$ was correct the points should be scatter around one, however this is not the pattern seen in the figure, rather the data follows a strict downward shape with increasing rotational speed. This points to an under estimation of the operational pressure at high rotational speed. It indicates that the prevailing method of excluding $k_{1}$ is a simplification of the rotating membrane system. 


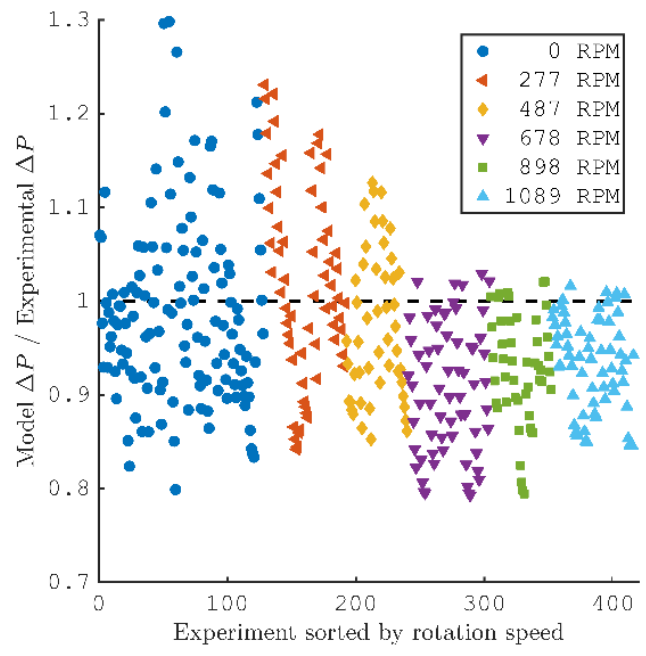

Figure 11: Residual plot sorted by rotational speed Residual plot of predicted $\Delta P$ over experimental $\Delta P$ using $k_{1}=1$ in Equation (eq: individual). The data was sorted according to the disc rotational speed, to show the under prediction at high rotational speeds.

To find a better value for the velocity factor, the experimental data was fitted to Equation 11 by the least squares method. The computed value of the velocity factor was fond to be $k_{1}=1.13$. In Figure 12 a parity plot of the experimental $\Delta P$ value plotted against $\Delta P$ value predicted by the fitted model. In the parity plot 353 out of 416 data points, i.e. $85 \%$ are within $10 \%$ of the experimental value. This shows the proposed model explains the data to a high degree.

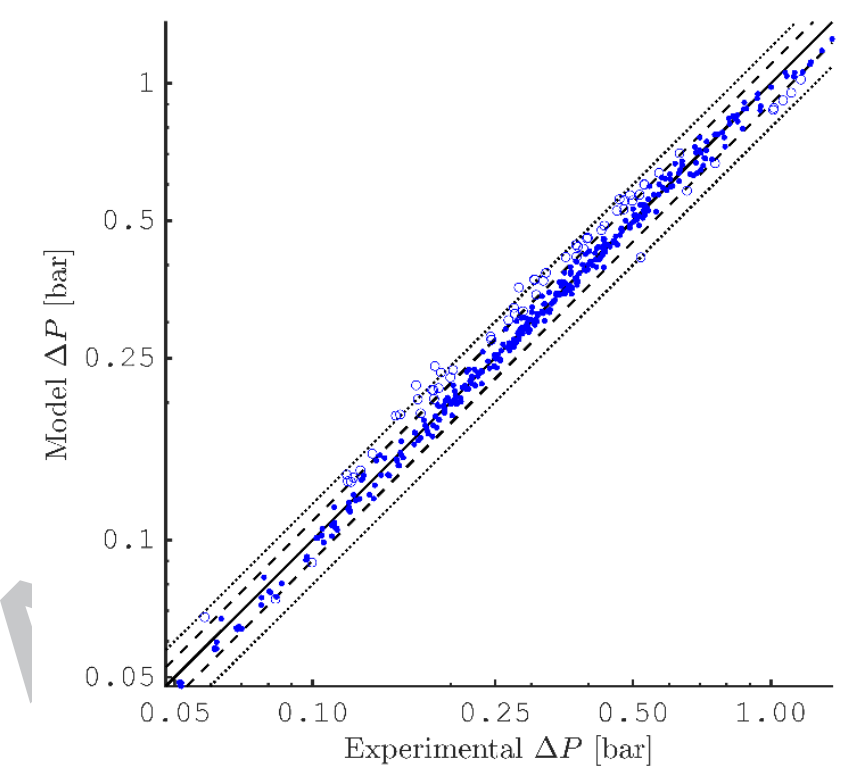

Figure 12: Log Log Parity plot, of Experimental $\Delta P$

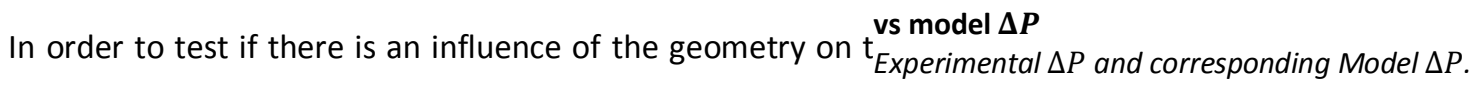
two, a collection with insert and a collection without inserDistance from solid centerline reflects deviation between model and measured data.

Equation 14, and the parameters determined. The procedurtDotted line: $20 \%$ deviation between data and to the used disc. The determined $k_{1}$ values are presented model value.

Dashed line: $10 \%$ deviation between data and

intervals. The values indicate that the shape of the module model value.

Filled dots falls within $10 \%$ deviation, open dots Ice factor. The installation of inserts in the module house seennot. 
pressure. The obtained $k_{1}$ values are however not statistically different. A more significant alteration to the module house would be needed to fully investigate this effect. As expected, the membrane disc was not found to be a significant factor in the magnitude of $k_{1}$.

The difference between a $k_{1}$ value of 1 and 1.13 results in a difference of the predicted counter pressure of 0.01 bar at 500 RPM, seemingly insignificant. However at 1500 RPM the difference in $P_{c}$ is 0.1 bar. For microfiltration systems this could be significant in relation to the TMP. Corrections for the counter pressure, as the one done in Equation 5 or Equation 6, could results in an under estimation of the corrected flux, leading to an unfavorable evaluation of the results when operating at high rotation.

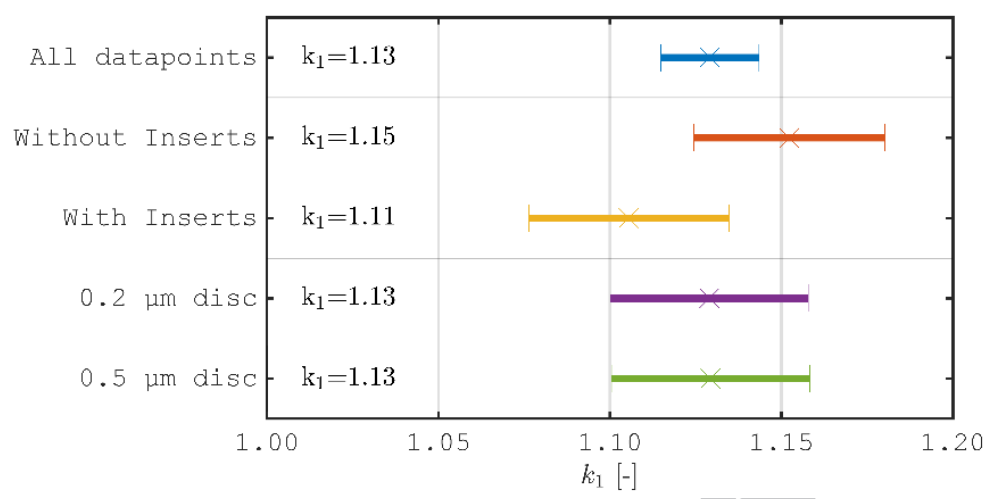

Figure 13: Computed velocity factors

Determined velocity factors $\left(k_{1}\right)$ with their $95 \%$ confidence interval.

The dataset have been separated according to the differences in geometry, i.e. insert, and the membrane disc used. The first collection has 416 data points, the remaining have 208 . 


\subsection{CFD Simulations}

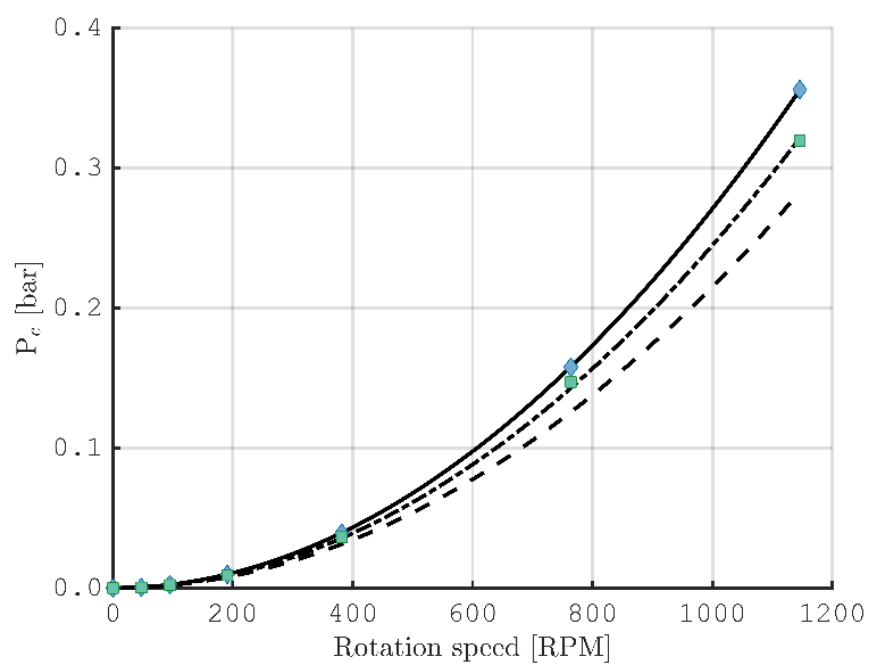

Figure 14: Simulation: Rotation vs Counter Pressure

Counter pressure in the simulated system.

$\checkmark$ : Simulation with insert. $\square$ : Simulation without insert.

Solid Line (-): Engineering model fitted on simulated systems with insert $\left(k_{1}=1.27\right)$.

Dotted Line (•-): Engineering model fitted on simulated systems without insert $\left(k_{1}=1.20\right)$.

Dashed Line (- $)$ : Engineering model fitted on experimental system $\left(k_{1}=1.13\right)$.

The CFD simulations of the system were evaluated at different rotational speeds. For each simulated case, the pressure data were extracted. By fitting Equation 10 to the simulated data, $k_{1}$ could be determined. In Figure 14 the data from the simulated systems are shown, together with the experimental model. In the figure simulated data from two different geometries are included, one for simulations without the insert, and one for simulations with the insert. In the CFD simulations rotation of the membrane also generates a counter pressure as seen in the experimental system. In the simulations the velocity factor was determined to $k_{1}=1.27$ for the geometry without insert and $k_{1}=1.20$ for the geometry with insert. Compared to the experimental value of $k_{1}=1.13$, this indicates that the effect is slightly stronger in the simulation, than in the real system. The discrepancies are between $10 \%$ and $15 \%$, depending on the geometry. In the simulated geometries the insert increases $k_{1}$, this is opposite of the effect hinted at in the experimental system, where the present of the insert reduced $k_{1}$. 


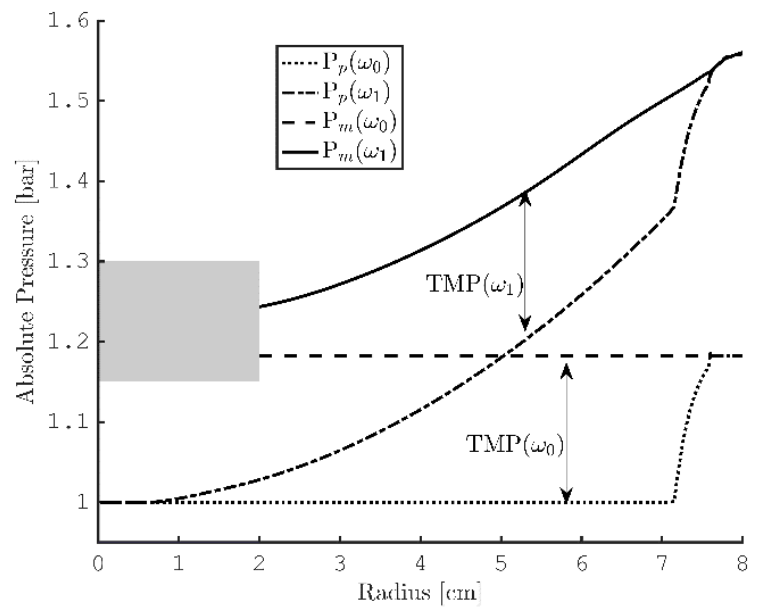

Figure 15: Pressure curves for membrane surface and permeate channel

Simulated pressure inside the permeate channel $\left(P_{P}\right)$ and at the membrane surface $\left(P_{m}\right)$ for a stationary $\left(\omega_{0}\right)$ and a spinning case $\left(\omega_{1}=\right.$ 1150 RPM). The difference in pressure profiles are a result of the counter pressure. The difference between $P_{m}$ and $P_{P}$ is the local TMP. The grey area is steel, thus there is not pressure data here.

At $r \approx 7.4 \mathrm{~cm}$ the end of the membrane starts influencing the TMP, hence the sharp decrease in TMP here.

Besides simulating the overall effect of the counter pressure, the simulations also gives an insight into the forces affecting the filtration locally within the membrane. One such local value is the local TMP, determined as the pressure at the membrane surface $\left(P_{m}\right)$ subtracted the pressure of the free permeate $\left(P_{p}\right)$, i.e. at the center of the membrane channel. In Figure 15 the radial dependency of $P_{m}$ and $P_{p}$ are shown for two very different rotational speeds, $\omega_{0}$ where the membrane is stationary and $\omega_{1}$ where the membrane rotates at high speed (1150 RPM). For the stationary membrane, both $P_{m}$ and $P_{p}$ is independent of the radial position, and TMP is therefore uniform for the length of the membrane. As the rotational speed increases both $P_{m}$ and $P_{p}$ takes on a pronounced slopped shape, as they both are affected by the counter rotational force. However the effect is not equal, the slope of $P_{p}$ is steeper, it is affect more by the rotation. In the simulations the total permeate flow is constant at all rotational speeds, therefore the overall driving force must remain constant. However locally it can change if it is compensated for elsewhere. Figure 16 shows the local TMP normalized by the system TMP for a stationary membrane. In the figure it can be seen, that high rotation leads to a higher local TMP at the inner edge, presumably to compensate for the lower local TMP at the outer edge. The shape of the local TMP curve corresponds to the local filtration performance, i.e. the driving force for filtration is lower at the outer edge of the membrane.

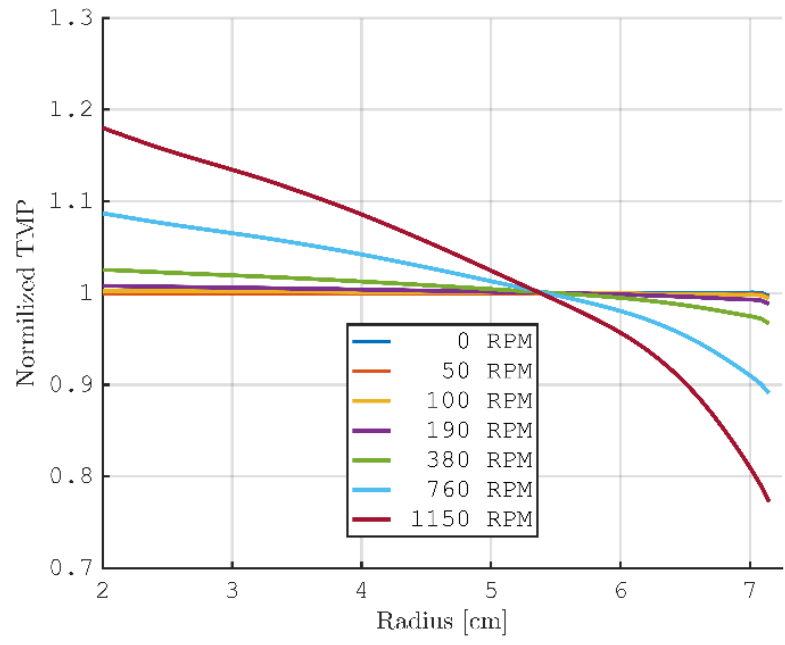

Figure 16: Normalized Local TMP Local TMP normalized by the system TMP for the stationary case. Shown as a function of position $(r)$ and rotational speed $(\omega)$. Higher rotation leads to higher local TMP at the inner edge, and lower local TMP at the outer edge. 


\section{Conclusion}

Based on 416 experiments it has been shown that the magnitude of the counter pressure present in dynamic filtration with rotating membrane can be explained by $P_{c}=\frac{\rho}{4} \cdot k_{1}^{2} \cdot\left(r_{1}^{2}+r_{2}^{2}\right) \cdot \omega^{2}$. Literature suggest $k_{1}=1$, but in this work experimental value of $k_{1}=1.13$ was determined. Additionally it was shown with CFD simulations that the value of $k_{1}$ exceed 1 in simulations. This highlight the need to quantify the systems counter pressure, before more complex experiments are conducted, similar to what is done for dynamic filtration systems with rotor. Likewise it has been shown, that water permeability can differ between experiments, despite including a cleaning step. Such observation implies that water permeability is not a constant, but should be measured before each experiments. Additionally a novel way to model membrane filtration in CFD has been shown. By defining the porosity and resistance as a function, a complex membrane structure could be reproduced without constructing a complicated computer geometry or simplifying the filtration system to a simple boundary condition. Based on experimental work there are weak indications, that there is a link between geometry and velocity factor, however more experiments are required to establish the extent of the effect.

\section{Acknowledgments}

This work is funded by the Innovation Fund Denmark (IFD) under File No. 5189-00189B and Novo Nordisk A/S

\section{References}

[1] B. Van der Bruggen, C. Vandecasteele, Distillation vs. membrane filtration: Overview of process evolutions in seawater desalination, Desalination. 143 (2002) 207-218. doi:10.1016/S00119164(02)00259-X.

[2] S.P. Beier, G. Jonsson, Separation of enzymes and yeast cells with a vibrating hollow fiber membrane module, Sep. Purif. Technol. 53 (2007) 111-118. doi:10.1016/j.seppur.2006.06.019.

[3] K.J. Hwang, S.J. Lin, Filtration flux-shear stress-cake mass relationships in microalgae rotating-disk dynamic microfiltration, Chem. Eng. J. 244 (2014) 429-437. doi:10.1016/j.cej.2014.01.076.

[4] L.H. Ding, M.Y. Jaffrin, M. Mellal, G. He, Investigation of performances of a multishaft disk (MSD) system with overlapping ceramic membranes in microfiltration of mineral suspensions, J. Memb. Sci. 276 (2006) 232-240. doi:10.1016/j.memsci.2005.09.051.

[5] M. Frappart, M.Y. Jaffrin, L.H. Ding, V. Espina, Effect of vibration frequency and membrane shear rate on nanofiltration of diluted milk, using a vibratory dynamic filtration system, Sep. Purif. Technol. 62 (2008) 212-221. doi:10.1016/j.seppur.2008.01.025.

[6] X. Xie, C. Le Men, N. Dietrich, P. Schmitz, L. Fillaudeau, Local hydrodynamic investigation by PIV and CFD within a Dynamic filtration unit under laminar flow, Sep. Purif. Technol. (2017). 
doi:10.1016/j.seppur.2017.04.009.

[7] G. Belfort, J.M. Pimbley, A. Greiner, K.Y. Chung, Diagnosis of membrane fouling using a rotating annular filter. 1. Cell culture media, J. Memb. Sci. 77 (1993) 1-22. doi:10.1016/0376-7388(93)85231-K.

[8] R. Bouzerar, L. Ding, M.Y. Jaffrin, Local permeate flux-shear-pressure relationships in a rotating disk microfiltration module: Implications for global performance, J. Memb. Sci. 170 (2000) 127-141. doi:10.1016/S0376-7388(99)00348-8.

[9] M.Y. Jaffrin, Dynamic shear-enhanced membrane filtration: A review of rotating disks, rotating membranes and vibrating systems, J. Memb. Sci. 324 (2008) 7-25. doi:10.1016/j.memsci.2008.06.050.

[10] R. Bouzerar, M.Y. Jaffrin, L. Ding, P. Paullier, Influence of geometry and angular velocity on performance of a rotating disk filter, AIChE J. 46 (2000) 257-265. doi:10.1002/aic.690460206.

[11] M.M. Dal-Cin, C.N. Lick, A. Kumar, S. Lealess, Dispersed phase back transport during ultrafiltration of cutting oil emulsions with a spinning membrane disc geometry, J. Memb. Sci. 141 (1998) 165-181. doi:10.1016/S0376-7388(97)00304-9.

[12] W. Zhang, L. Ding, N. Grimi, M.Y. Jaffrin, B. Tang, Application of UF-RDM (Ultafiltration Rotating Disk Membrane) module for separation and concentration of leaf protein from alfalfa juice: Optimization of operation conditions, Sep. Purif. Technol. 175 (2017) 365-375. doi:10.1016/j.seppur.2016.11.059.

[13] Y. Taamneh, S. Ripperger, Performance of single and double shaft disk separators, Phys. Sep. Sci. Eng. 2008 (2008). doi:10.1155/2008/508617.

[14] M. Mulder, Basic principles of membrane technology, Kluwer Academic, 1996. http://findit.dtu.dk/en/catalog/2304901555 (accessed November 21, 2017).

[15] J. Luo, Z. Zhu, L. Ding, O. Bals, Y. Wan, M.Y. Jaffrin, E. Vorobiev, Flux behavior in clarification of chicory juice by high-shear membrane filtration: Evidence for threshold flux, J. Memb. Sci. 435 (2013) 120-129. doi:10.1016/j.memsci.2013.01.057.

[16] M.Y. Jaffrin, G. He, L.H. Ding, P. Paullier, Effect of membrane overlapping on performance of multishaft rotating ceramic disk membranes, Desalination. 200 (2006) 269-271. doi:10.1016/j.desal.2006.03.341. 


\section{Highlights}

- The counter pressure found in rotational dynamic filtration was investigated

- An experimental system was evaluated with different liquid systems

- The counter pressure can be described by the velocity factor

- CFD simulation was performed to refine understanding of velocity factor

- Confirmation that the velocity factor is higher than the theoretical value of 1 